Article

\title{
Orally Administrated Recombinant Vaccinia Virus Displaying ROP4 Induces Protection against Toxoplasma gondii Challenge Infection
}

\author{
Keon-Woong Yoon ${ }^{1,+}{ }^{+}$, Ki-Back Chu ${ }^{2,3}$, Hae-Ji Kang ${ }^{1}$, Min-Ju Kim ${ }^{1}$, Gi-Deok Eom ${ }^{1}$ and Fu-Shi Quan ${ }^{2,3, *(D)}$ \\ 1 Department of Biomedical Science, Graduate School, Kyung Hee University, Seoul 02447, Korea; \\ ky20200310318@khu.ac.kr (K.-W.Y.); heajik0514@khu.ac.kr (H.-J.K.); mj16441@khu.ac.kr (M.-J.K.); \\ ekd3910@khu.ac.kr (G.-D.E.) \\ 2 Department of Medical Zoology, School of Medicine, Kyung Hee University, \\ Seoul 02447, Korea; kbchu@khu.ac.kr \\ 3 Medical Research Center for Bioreaction to Reactive Oxygen Species and Biomedical Science Institute, \\ School of Medicine, Graduate School, Kyung Hee University, Seoul 02447, Korea \\ * Correspondence: fsquan@khu.ac.kr \\ + These authors contributed equally to this work.
}

check for

updates

Citation: Yoon, K.-W.; Chu, K.-B.;

Kang, H.-J.; Kim, M.-J.; Eom, G.-D.; Quan, F.-S. Orally Administrated Recombinant Vaccinia Virus

Displaying ROP4 Induces Protection against Toxoplasma gondii Challenge Infection. Vaccines 2022, 10, 152.

https://doi.org/10.3390/

vaccines 10020152

Academic Editor: Ingo Drexler

Received: 30 November 2021

Accepted: 18 January 2022

Published: 20 January 2022

Publisher's Note: MDPI stays neutral with regard to jurisdictional claims in published maps and institutional affiliations.

Copyright: () 2022 by the authors Licensee MDPI, Basel, Switzerland. This article is an open access article distributed under the terms and conditions of the Creative Commons Attribution (CC BY) license (https:// creativecommons.org/licenses/by/ $4.0 /)$.

\begin{abstract}
Recombinant vaccinia viruses (rVVs) are attenuated viruses and are widely utilized as vectored vaccine platforms against numerous diseases. However, the protective efficacy of these rVV vaccines against Toxoplasma gondii and the resulting mucosal immunity has not been thoroughly assessed. Here, rVVs expressing the rhoptry protein 4 (ROP4) of T. gondii were generated. To evaluate the protection induced by the vaccines, mice were orally immunized with the ROP4-rVVs and subsequently challenge-infected with a lethal dose of T. gondii ME49 strain. Immunization with the rVVs induced higher levels of parasite-specific IgG and IgA antibody responses in sera compared to unimmunized control (NC). Upon challenge infection, significantly higher levels of $\operatorname{IgG}$ or $\operatorname{IgA}$ antibody responses in the brain, intestines, and vaginal samples were found in the immunized mice compared to NC. The ROP4-rVV vaccination elicited potent IgG and IgA secreting cell (ASC) responses, while substantially enhancing germinal center $\mathrm{B}$ cell, as well as $\mathrm{CD}^{+}$and $\mathrm{CD} 8^{+} \mathrm{T}$ cell responses from lymphoid organs. The production of pro-inflammatory cytokines IFN- $\gamma$ and IL- 6 in the brains was markedly diminished following immunization. The immunized mice also experienced reduced bodyweight loss and possessed fewer brain cysts than the control group. These results suggest that oral delivery of ROP4 displaying rVVs induced mucosal and systemic immunities that contributed to protection against lethal T. gondii challenge infection.
\end{abstract}

Keywords: recombinant vaccinia virus; ROP4; T. gondii; vaccine; ME49; mucosal immunity

\section{Introduction}

Infection in humans can occur through the ingestion of undercooked meat and contaminated water, resulting in severe symptoms depending on the host's immune status. In the case of immunocompromised individuals, pregnant women, and the elderly, it can cause congenital toxoplasmosis, resulting in hydrocephalus and microcephaly, and in severe cases miscarriage [1]. The current treatment options for T. gondii infection involve co-administration of pyrimethamine and sulfadiazine. Other drugs such as hydroxynaphthoquinone (atovaquone) and azithromycin could also be used, but there are major issues with these drugs. Pathogen drug resistance is a recurring theme frequently reported from various diseases and toxoplasmosis is no exception. This and the severe side effects, poor patient tolerability, and therapeutic ineffectiveness against bradyzoites of $T$. gondii are several of the obstacles that must be surmounted [1]. Vaccines are widely considered to be the most cost-effective healthcare intervention, and successful vaccines can benefit public 
health, which ultimately translates to economic growth [2]. For these reasons, developing a vaccine to protect against toxoplasmosis is an urgent need.

Vaccinia virus has been used extensively as a vaccine platform against numerous diseases such as smallpox. Currently, genetically modified vaccinia virus-based vaccines such as the modified vaccinia Ankara (MVA) are frequently used in heterologous primeboost immunization strategies and have proven to be highly effective. To date, only a few studies have investigated the vaccine efficacies of recombinant vaccinia virus against toxoplasmosis. Roque-Resndiz et al. [3] reported that ROP2-expressing MVA vaccines were capable of inducing T. gondii-specific antibodies and that their immunization prolonged the survival of mice upon challenge infection with the RH strain. Heterologous immunization strategies involving MVA vaccines were also successful. In comparison to homologous immunization, heterologously immunizing GRA4-expressing DNA and MVA vaccines elicited greater brain cyst reduction and promoted longer survival of immunized mice after challenge infecting 20,000 PLK strains [4]. Similar findings were observed in another comparative study. Heterologously immunizing mice with SAG1-expressing adenovirus and MVA vaccines induced greater protection than homologous adenovirus-vectored vaccines [5].

The rhoptry proteins of $T$. gondii are parasitic components required for proliferation and host cell invasion [6,7]. Several studies have assessed the efficacy of ROP4 antigens using various vaccine platforms such as virus-like particles (VLPs) [8] or subunit vaccines [9]. Furthermore, we have recently investigated the efficacy of ROP4-expressing recombinant baculovirus (rBV) vaccines and the resulting immune responses in mice [10]. Nevertheless, there are inherent problems with the baculovirus-based vaccines. First and foremost, repeated vaccination with the baculovirus vaccines can result in the development of baculovirus-specific antibodies in hosts and subsequent neutralization of the baculovirus upon re-entry [11,12]. Another issue is the rapid degradation of baculoviral particles by the host complement system, thus limiting their application as vaccines $[12,13]$. To address these limitations of our previous works, we generated a ROP4-expressing recombinant vaccinia virus (rVV) vaccine as not a single study has investigated their efficacy in animal models. Orally administered vaccines are highly sought after due to their mucosal and systemic immunity-inducing effects [14]. Given that T. gondii is orally acquired in hosts, mucosal immunity is of importance since the intestinal mucosa acts as the initial site of infection for T. gondii and many other pathogens. Here, we investigated the resulting mucosal immunity induced by the ROP4-rVVs against $T$. gondii infection, which has yet to be reported. Our findings demonstrated that the ROP4-rVV vaccines elicited a robust cellular and humoral response that protected mice upon lethal T. gondii infection.

\section{Materials and Methods}

\subsection{Animals and Ethics}

Six-week-old female BALB/c mice purchased from NARA Biotech (Seoul, Korea) were used for this study. The animals were housed in a specific-pathogen-free facility with easy access to food and water. All animal experiment protocols were approved and performed in accordance with the guidelines of Kyung Hee University IACUC (permit number: KHUASP (SE) 20-648).

\subsection{Parasite, Cells, and Antibodies}

Parasites and cells used in this study were maintained as described earlier (Kang et al., 2020b). T. gondii ME49 strain was maintained by serial passaging in BALB/c mice. Mice were sacrificed and ME49 cysts were purified from the brain tissues of infected mice. CV-1 and Vero cells were cultured in T175 flasks with Dulbecco's Modified Eagle's Medium (ThermoFisher, Waltham, MA, USA) supplemented with $1 \%$ penicillin/streptomycin and $10 \%$ fetal bovine serum (FBS) at $37^{\circ} \mathrm{C}$. Horseradish peroxidase (HRP)-conjugated goat anti-mouse IgG and IgA secondary antibodies were purchased from Southern Biotech (Birmingham, AL, USA). 


\subsection{Generation of Recombinant Vaccinia Virus ( $r V V)$}

The rhoptry protein 4 (ROP4) gene was amplified by polymerase chain reaction (PCR) and cloned into pRB21 vectors with StuI/HindIII restriction enzymes [15]. Prior to transfection with the recombinant $\mathrm{pRB} 21$ plasmid containing the ROP4 gene using Cellfectine II reagent (Invitrogen, Waltham, MA, USA), CV-1 cells were infected with the nonplaque-forming vRB12 mutant as previously described [16]. Infected cells were harvested with a scraper once morphological changes and other cytopathic effects were detected from approximately $70 \%$ of the seeded cells and centrifuged to pellet the cells. Supernatants were aspirated and cells were subjected to repeated freeze-thaw cycles that involved freezing at $-80{ }^{\circ} \mathrm{C}$ in a deep freezer for 30 min immediately followed by thawing at $37^{\circ} \mathrm{C}$ in a water bath a total of 3 times. After this procedure, cells were sonicated and centrifuged at $6000 \mathrm{rpm}, 20 \mathrm{~min}, 4^{\circ} \mathrm{C}$. Supernatants were removed and pellets were resuspended in fresh DMEM media. To further purify the rVVs, resuspended rVV-containing cell pellets were carefully stacked on top of $36 \%$ sucrose gradient. After centrifugation at 20,000 rpm, $30 \mathrm{~min}, 4^{\circ} \mathrm{C}$, a distinct clear band was observed and this fraction was carefully collected. After resuspending the band fraction containing the rVV in 0.1 M PBS, protein concentration was determined using the QuantiPro BCA Assay Kit (Sigma-Aldrich, St Louis, MA, USA).

\subsection{Quantification of $r V V$ s by Plaque Assay}

Vero cells were used to quantify rVV titers. In brief, confluent monolayers of Vero cells were cultured in 12-well plates in DMEM media supplemented with $1 \%$ penicillin/streptomycin and $10 \%$ FBS. Upon reaching $90-100 \%$ confluence, cells were infected with the serially diluted $\mathrm{rVVs}\left(10^{2}, 10^{3}, 10^{4}\right.$ dilutions in serum-free media) and incubated at $37^{\circ} \mathrm{C}, 5 \% \mathrm{CO}_{2}$ for $1 \mathrm{~h}$. Virus inoculum was removed after $1 \mathrm{~h}$ and overlay media (1\% noble agar and $2 \%$ FBS in DMEM media) were gently added into each well. Plates were incubated at $37^{\circ} \mathrm{C}$ for 4 days and enlarged viral plaques were counted to determine rVV titers.

\subsection{Immunization and Challenge Infection}

A total of 18 mice were subdivided into 3 groups ( $n=6$ per group): unimmunized (Naïve), unimmunized mice that were challenge-infected (Naïve+Cha), and rVVimmunized mice. Mice were primed and boost-immunized with $5 \times 10^{3} \mathrm{pfu} / 100 \mu \mathrm{L}$ of ROP4-rVVs through the oral route by oral gavage at 4-week intervals. Four weeks after the boost immunization, mice were orally challenge-infected with a $50 \mathrm{LD}_{50}$ dose (2000 cysts) of ME49 cysts. Mice were monitored daily to assess changes in bodyweight and survival rates. Mice that lost more than $20 \%$ of their initial bodyweight were humanely euthanized. At 16 days post-infection (dpi), all the mice were sacrificed for sample collection and immunological assays were performed.

\subsection{Sample Collection and Preparation}

Samples were collected as previously described [10]. Four weeks after each immunization, blood samples of mice were collected by retro-orbital plexus puncture. Sera were separated from the blood via centrifugation at 5000 RPM for $10 \mathrm{~min}$. Sera were diluted (1:100 dilution in PBS) and used as primary antibodies for ELISA. After sacrificing all of the mice at $16 \mathrm{dpi}$, brain tissues and other mucosal samples were acquired. Briefly, $100 \mu \mathrm{L}$ of $0.1 \mathrm{M}$ PBS was added per $0.1 \mathrm{~g}$ of fecal sample. Vaginal samples were collected by washing the vaginal canals with $200 \mu \mathrm{L}$ of $0.1 \mathrm{M}$ PBS. Intestinal samples were harvested by longitudinally sectioning the intestines and immersing the tissues in $500 \mu \mathrm{L}$ of $0.1 \mathrm{M}$ PBS. Mucosal samples were incubated at $37^{\circ} \mathrm{C}$ for $1 \mathrm{~h}$ then centrifuged at $500 \mathrm{rpm}$ for $10 \mathrm{~min}$. Supernatants were collected and samples were stored at $-20^{\circ} \mathrm{C}$ until use. The brain tissues were used to quantify cyst burden in mice. Spleens and mesenteric lymph nodes were carefully harvested for immunological assays. All the samples were processed on an individual basis. 


\subsection{Enzyme-Linked Immunosorbent Assay (ELISA) and Antibody-Secreting Cell (ASC) Responses}

T. gondii-specific serum antibody responses were determined as previously described $[8,10]$. To evaluate ASC induction, splenic and mesenteric lymph nodes (MLNs) were collected from mice. Single cell suspensions of splenocytes and MLN cells were prepared as previously described [8]. After RBC lysis, splenocytes and MLN cells were seeded into 96-well immunoplates coated with T. gondii ME49 whole antigen $(4 \mu \mathrm{g} / \mathrm{mL})$. Plates were incubated for 5 days at $37^{\circ} \mathrm{C}$. Supernatants were removed and HRP-conjugated anti-mouse IgG or IgA antibodies were added into each well. Plates were incubated for $1 \mathrm{~h}$ at $37^{\circ} \mathrm{C}$, and 100 $\mu \mathrm{L}$ of OPD substrate dissolved in citrate substrate buffer were inoculated into each well. Reactions were stopped using dilute $\mathrm{H}_{2} \mathrm{SO}_{4}$ and $\mathrm{OD}_{490}$ values were measured.

\subsection{Flow Cytometry and Immune Cell Population Assessment}

Flow cytometry was performed on splenocytes and MLN cells as previously described [17]. Splenocytes $\left(1 \times 10^{6}\right.$ cells/mouse $)$ and MLN cells $\left(1 \times 10^{5}\right.$ cells/mouse $)$ were stimulated with T. gondii ME49 antigen $(2 \mu \mathrm{g} / \mathrm{mL})$ at $37^{\circ} \mathrm{C}$ with $5 \% \mathrm{CO}_{2}$ for $2 \mathrm{~h}$. Stimulated cells were stained with fluorophore-conjugated antibodies purchased from BD Biosciences (Franklin Lakes, NJ, USA) and Invitrogen (Waltham, MA, USA). CD4 ${ }^{+} \mathrm{T}$ cells, CD8 ${ }^{+} \mathrm{T}$ cells, and germinal center $\mathrm{B}$ (GC B) cell populations were measured using the following antibodies: CD3 (PE- Cy7), CD4 (FITC), CD8 (PE), GL7 (PE), and B220 (FITC). All staining procedures were performed according to the manufacturer's protocol. Stained cells were acquired by Accuri $\mathrm{C} 6$ flow cytometer and analyzed with the C6 Accuri software (BD Biosciences, Franklin Lakes, NJ, USA). For GC B cells, B220 lung cells and splenocyte populations were gated, and from this population those positive for GL7 were gated. For $\mathrm{CD}^{+}$and $\mathrm{CD}^{+}{ }^{+}$cells, CD3-positive single cell populations were gated and from this population $\mathrm{CD}^{+}$and $\mathrm{CD} 8^{+}$cells were determined.

\subsection{Inflammatory Cytokine Production and Cyst Burden in the Brain}

Brain tissues of individual mice were subdivided into left and right hemispheres and processed separately for inflammatory cytokine assessment and cyst count, respectively. The left hemispheres were homogenized in $500 \mu \mathrm{L}$ of PBS and then centrifuged at $10,000 \mathrm{rpm}$ for $10 \mathrm{~min}$. Supernatants were carefully collected and stored at $-80^{\circ} \mathrm{C}$ until use. Pro-inflammatory cytokines in these supernatants were measured using IFN- $\gamma$ and IL-6 BD OptEIA ELISA kits (BD Biosciences, Franklin Lakes, NJ, USA). All experiments were performed according to the manufacturer's instructions and standard curves were generated to calculate the cytokine concentrations. The right hemispheres of the brains were used to quantify cyst burdens in challenge-infected mice following the method previously described [18]. Tissues were homogenized in $500 \mu \mathrm{L}$ of PBS and centrifuged at 12,100 rpm for $20 \mathrm{~min}$ through Percoll density gradient media (BD Biosciences, Franklin Lakes, NJ, USA). The T. gondii cyst layer was carefully collected and repeatedly washed with PBS. Cysts from each sample were counted 3 times from 3 different fields of view under a microscope (Leica DMi8, Leica, Wetzlar, Germany).

\subsection{Statistical Analysis}

All statistical analyses were performed using Graphpad Prism version 6 (San Diego, CA, USA). A one-way ANOVA with Tukey's post hoc test or 2-way ANOVA with Bonferroni's post hoc test was performed to assess the statistical significance between the means. Data are presented as mean $\pm \mathrm{SD}$ and statistical significance is denoted with asterisks. $p$ values of ${ }^{*} p<0.05,{ }^{* *} p<0.01,{ }^{* * *} p<0.001$, and ${ }^{* * * *} p<0.0001$ were considered statistically significant.

\section{Results}

\subsection{Vaccinia Virus Characterization}

The T. gondii ROP4 gene was PCR-amplified and subsequently cloned into the pRB21 vector. To confirm the successful integration of the ROP4 gene into the pRB21 vector, 
restriction enzyme digestion with StuI and HindIII was performed (Figure 1A). Virus titers of the rVVs were determined by plaque assay. Distinct white plaques were detected from the Vero cells infected with the serially diluted $\mathrm{rVVs}\left(10^{2}, 10^{3}, 10^{4}\right.$ dilutions). Plaque counts were inversely proportional to the dilution fold, with the highest number of plaques being observed from $10^{2}$ dilutions. No plaques were observed from the vehicle control group (Figure 1B).
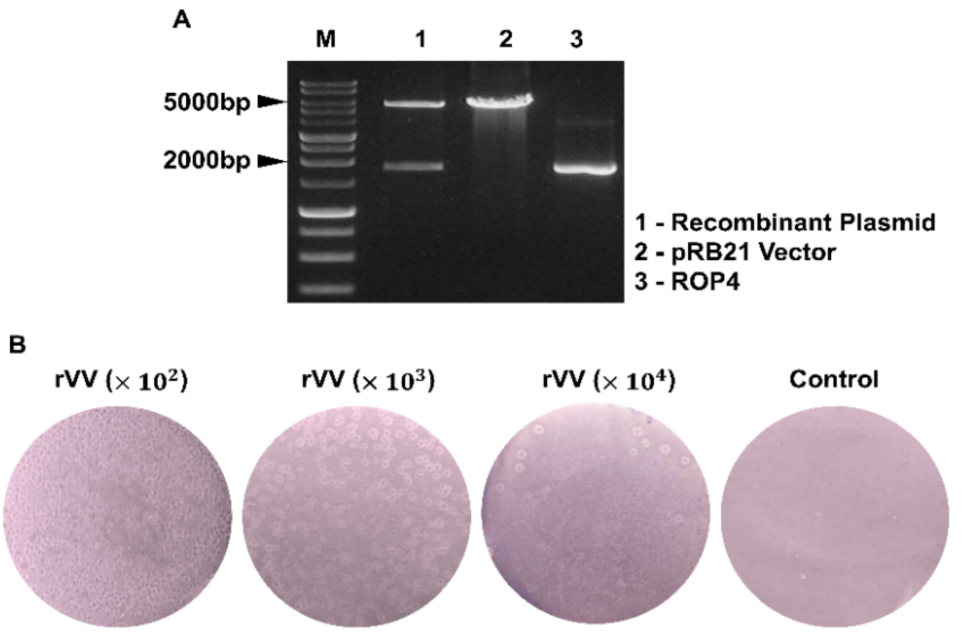

Figure 1. Recombinant vaccinia virus characterization. Recombinant plasmid constructs containing the T. gondii ROP4 gene were cleaved with the restriction enzymes. The size of the ROP4 gene and the pRB21 vectors were 1761 bp and 5537 bp, respectively (M: Marker, Lane 1: pRB21 + ROP4, Lane 2: pRB21, Lane 3: ROP4) (A). Virus titers of the ROP4-rVV were determined by plaque assay in Vero cells. Plaques were visualized under a microscope at 4 dpi. Representative images from 3 independent experiments performed in triplicates were acquired at $100 \times$ magnification $(\mathbf{B})$.

\subsection{IgG and IgA Antibody Response in Sera and Mucosal Samples}

The T. gondii-specific antibody responses were measured using the sera acquired from mice after prime and boost immunizations. Compared to unimmunized control, immunization with the ROP4-rVV elicited antibody responses. Specifically, increasing the number of immunizations enabled greater production of both IgG and IgA in the sera. In stark contrast to the IgG responses, a drastic increase in IgA response was observed after boost immunization (Figure 2A,B). At $16 \mathrm{dpi}$, mucosal tissue samples were harvested and antibody responses were assessed. Similar to the serum ELISA data acquired after the two immunizations, antibody inductions in the mucosal tissues were also observed after the challenge infection. Compared to the Naïve+Cha group, significant differences in brain IgG and IgA levels were detected (Figure 2C,D). An identical trend was also observed from the intestinal samples, which was characterized by robust IgG and IgA responses from the immunized mice (Figure 2E,F). Marginal increases in antibody responses were also detected from the vaginal secretions of mice (Figure 2G,H). Expectedly, mucosal antibody responses were induced by oral immunization with ROP4-rVVs in mice.

\subsection{Antibody Secreting Cell Response}

To determine antibody-secreting cell responses, splenocytes and MLN cells were collected from mice and cultured for 5 days with T. gondii antigens. Compared to both unimmunized control and Naïve+Cha mice, splenic IgG ASC responses were significantly higher in the immunized mice (Figure 3A). While this was also the case with splenic IgA ASC, the differences between the means of Naïve+Cha and ROP4-rVV were not as drastic as those of IgG (Figure 3B). ASC responses in the MLN were similar to those demonstrated by splenocytes. Immunization with the rVVs elicited significantly greater quantities of $\operatorname{IgG}$ and IgA ASC responses than Naïve+Cha (Figure 3C,D). 

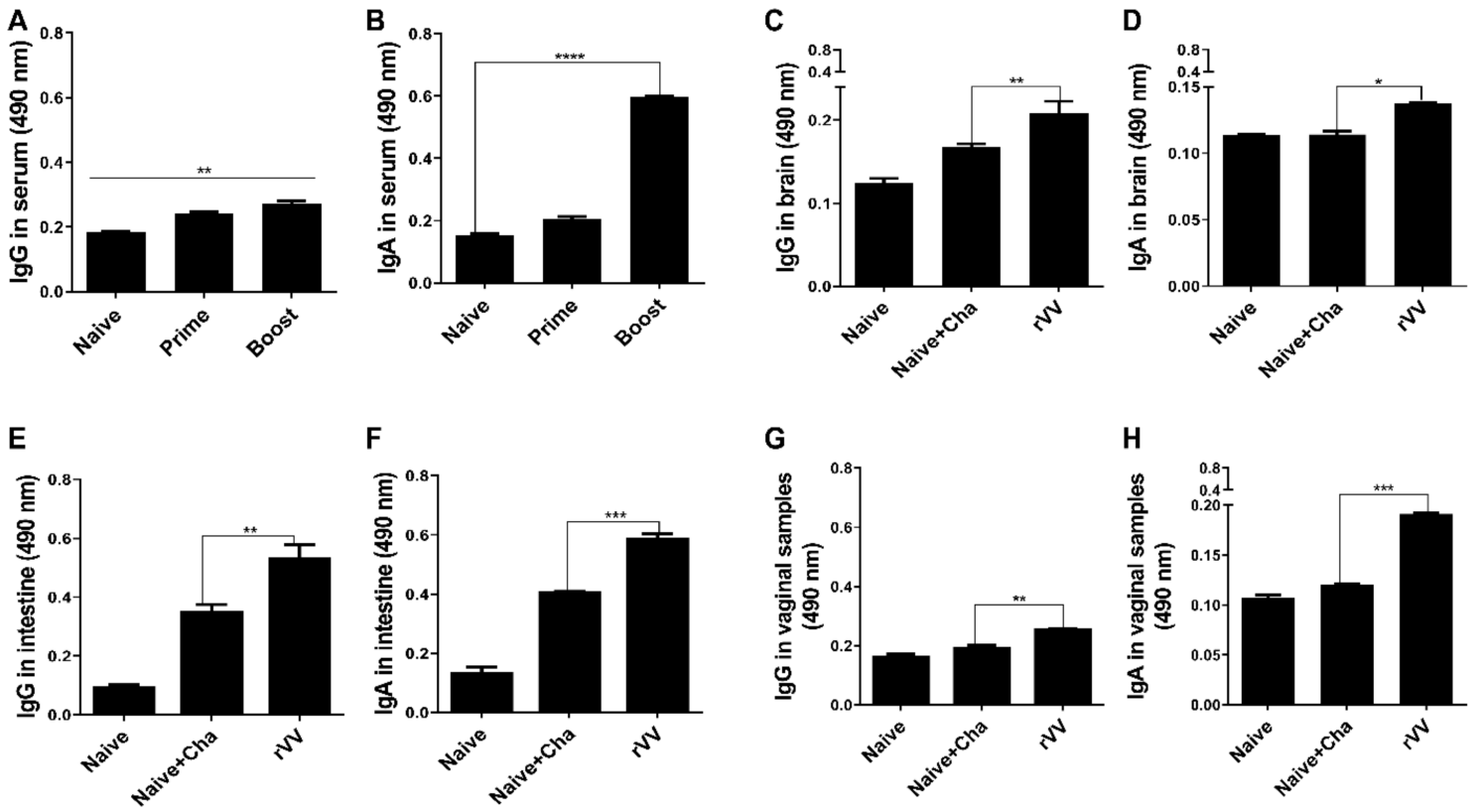

Figure 2. Evaluating serum and mucosal antibody inductions. BALB/C mice ( $n=6$ per group) were immunized with the ROP4-rVVs by the oral route. Sera were collected 4 weeks after immunization, and T. gondii-specific sera IgG and IgA responses to T. gondii ME49 antigen were determined by ELISA (A,B). T. gondii-specific mucosal IgG and IgA responses in the brain (C,D), intestines (E,F), and vaginal secretions $(\mathbf{G}, \mathbf{H})$ were collected at $16 \mathrm{dpi}$ to evaluate mucosal antibody responses. Data are presented as mean \pm SD from 3 independent experiments performed in triplicates and asterisks indicate statistical differences between groups. $\left({ }^{*} p<0.05,{ }^{* *} p<0.01,{ }^{* * *} p<0.001,{ }^{* * * *} p<0.0001\right.$ ).

A

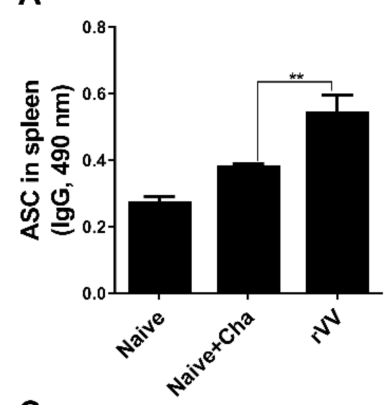

C

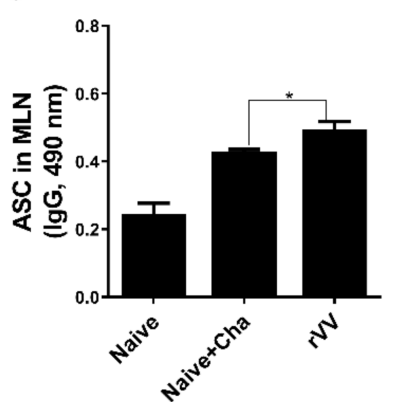

B

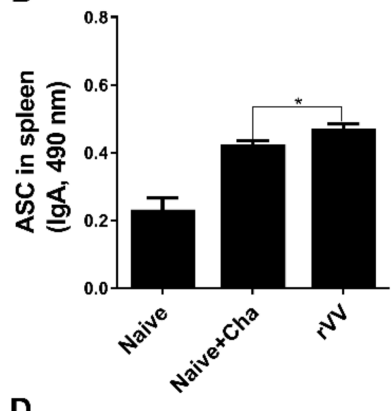

D

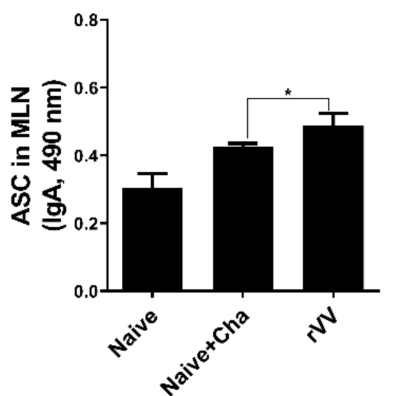

Figure 3. Antibody-secreting cell responses in secondary lymphoid organs. At 16 dpi, mice ( $n=6$ per group) were sacrificed and the levels of IgG and IgA antibody-secreting cells were evaluated in spleen and MLN cells. Single-cell populations of splenocytes and MLN cells were incubated with T. gondii ME49 lysate antigen. Splenic IgG and IgA (A,B, respectively) and MLN IgG and IgA (C,D, respectively) were evaluated by ELISA. Data are presented as mean \pm SD from 3 independent experiments performed in triplicates and asterisks indicate statistical differences between groups. $\left({ }^{*} p<0.05,{ }^{* *} p<0.01\right)$. 


\subsection{Germinal Center B Cell Response in Spleen and MLN}

Vaccine-induced immune responses were further assessed using flow cytometry. At $16 \mathrm{dpi}$, compared to Naïve+Cha control group, splenic GC B cell populations were nearly doubled in the ROP4-rVV immunization group (Figure 4A). An identical trend was also observed with MLN as well (Figure 4B). A scatterplot depicting GC B cell populations from both splenocytes and MLN (Figure 4C,D) revealed that ROP4-rVV immunization contributed to high levels of GC B cell induction in both lymphoid organs.
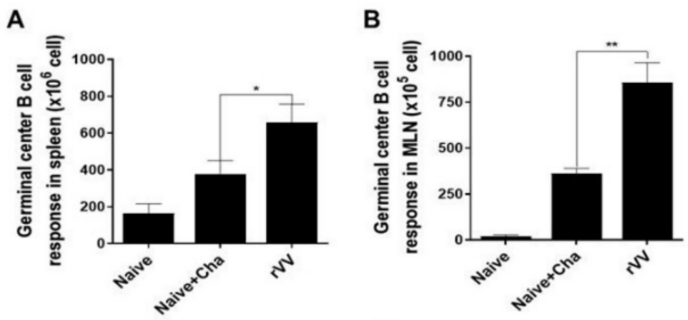

c

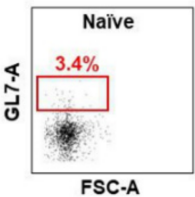

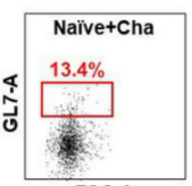

FSC-A

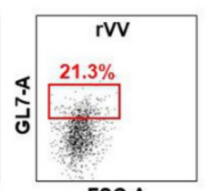

FSC-A
D

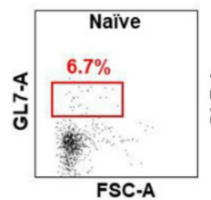

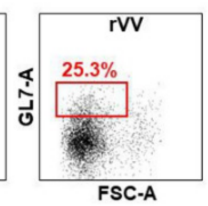

Figure 4. ROP4-rVV immunization induces potent GC B cell responses. Spleens and MLNs were harvested from mice ( $n=6$ per group) at $16 \mathrm{dpi}$ and GC B cell proliferation was assessed by flow cytometry. GC B cell populations from antigen-stimulated spleen (A) and MLN (B) were evaluated. Representative scatterplots for both spleen (C) and MLN (D) were provided, with boxes indicating the gated GC B cell population. Data are presented as mean \pm SD from 3 independent experiments performed in triplicates and asterisks denote statistical differences between groups $\left({ }^{*} p<0.05,{ }^{* *} p<0.01\right)$.

\subsection{Activation of $\mathrm{CD}^{+}$and $\mathrm{CD}^{+} \mathrm{T}$ Cells in $\mathrm{MLN}$}

$\mathrm{CD}^{+}$and $\mathrm{CD}^{+} \mathrm{T}$ cell activity was measured in mesenteric lymph nodes. Drastically increased $\mathrm{CD}^{+} \mathrm{T}$ cell populations were observed from Naïve+Cha and ROP4-rVV immunization groups, with $\mathrm{CD}^{+} \mathrm{T}$ cell induction being significantly greater in the latter (Figure 5A). Profound $\mathrm{CD}^{+} \mathrm{T}$ cell proliferation was also detected from the MLNs of Naïve+Cha and ROP4-rVV groups (Figure 5B). Gated T cell populations for each group were illustrated using a scatterplot (Figure 5C). Representative plots reveal that challenge infection with the $50 \mathrm{LD}_{50} \mathrm{~T}$. gondii ME49 enhanced $\mathrm{CD}^{+}$and $\mathrm{CD}^{+} \mathrm{T}$ cell populations by roughly $30 \%$ and $12 \%$, respectively. These values were further enhanced if the mice were immunized with the ROP4-rVVs.

\subsection{Pro-Inflammatory Cytokine Response}

To determine the amount of pro-inflammatory cytokines, IFN- $\gamma$ and IL- 6 levels in the brain homogenates were measured. Compared to the Naïve control, large quantities of inflammatory cytokines were produced in the brain tissues of Naïve+Cha mice. Vaccination with the ROP4-rVV ameliorated the inflammatory response by significantly reducing the production of IFN- $\gamma$ (Figure 6A) and IL-6 (Figure 6B) in the brains of mice. 
A

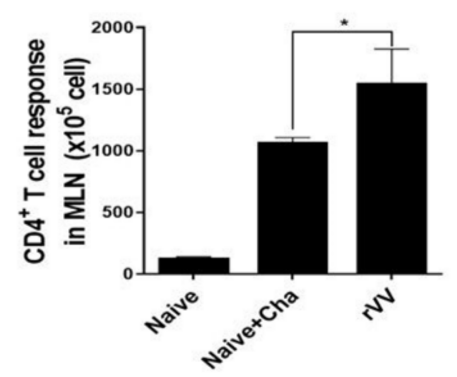

C

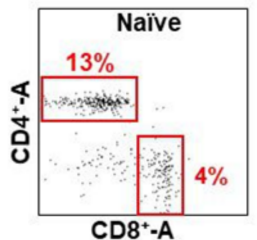

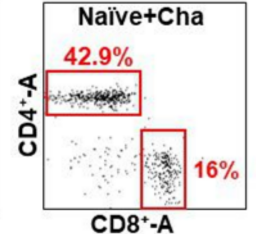

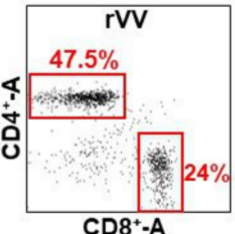

Figure 5. ROP4-rVV enhances T cell inductions in the MLNs of immunized mice. MLN cells of mice were collected. Single-cell populations of MLN cells were stimulated with T. gondii ME49 antigens. $\mathrm{CD}^{+}(\mathrm{A})$ and $\mathrm{CD}^{+}(\mathbf{B}) \mathrm{T}$ cells were quantified using flow cytometry. Representative scatterplots illustrating lymphocyte gating of the $\mathrm{CD}^{+}$and $\mathrm{CD}^{+} \mathrm{T}$ cells from 3 independent experiments were provided $(\mathbf{C})$. Boxes indicate the percentages of both $\mathrm{CD} 4^{+}$and $\mathrm{CD} 8^{+} \mathrm{T}$ cells. Data are presented as mean \pm SD from 3 independent experiments and asterisks denote statistical differences between groups $(* p<0.05)$.

A

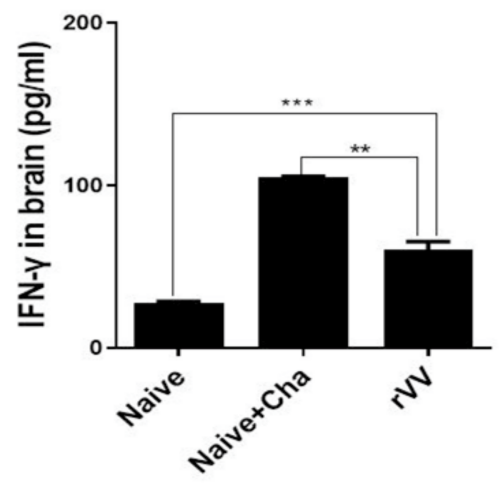

B

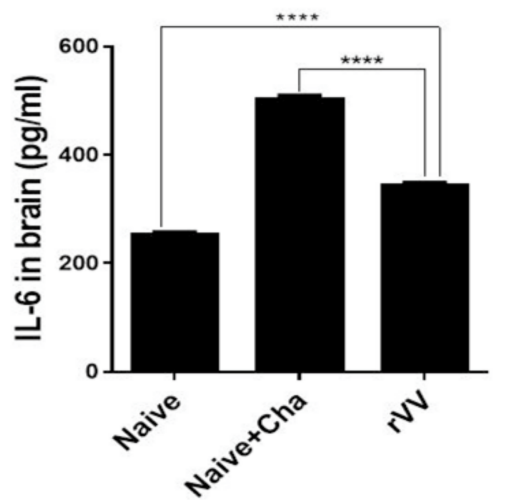

Figure 6. Immunization with ROP4-rVV vaccine inhibits inflammatory cytokine production. Brain tissues were isolated from mice and tissue homogenates were used to detect cytokines IFN- $\gamma(\mathbf{A})$ and IL-6 (B) by ELISA. Data are presented as mean \pm SD from 3 independent experiments performed in triplicates and asterisks indicate statistical differences between groups ${ }^{* *} p<0.01,{ }^{* * *} p<0.001$, **** $p<0.0001)$.

\subsection{Protective Efficacy of the ROP4- $r$ VV Vaccine}

To confirm the protective efficacy of ROP4-rVV against T. gondii ME49 infection, brain cysts were counted under the microscope. More than 6000 cysts were counted in Naïve+Cha, whereas the immunized mice had approximately a threefold decrease in the number of parasitic cysts (Figure 7A). All Naïve+Cha mice lost more than $20 \%$ of their initial bodyweight and were humanely euthanized at $16 \mathrm{dpi}$ with a lethal dose of ME49. Contrary to these results, ROP4-rVV immunization ensured that mice maintained bodyweights close to the normal range (Figure 7B). Although all of the Naïve+Cha mice perished, none of the immunized mice showed signs of illness, and $100 \%$ survival was 
observed (Figure 7C). These results indicate that administering the ROP4-rVV vaccine via the oral route is effective against T. gondii ME49 infection.

A

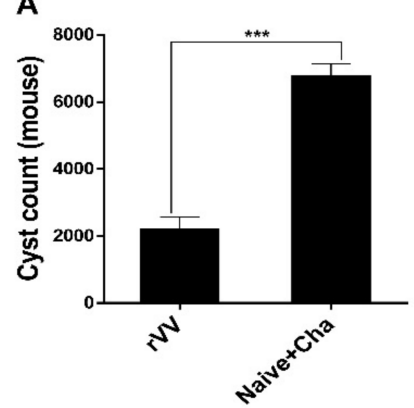

B

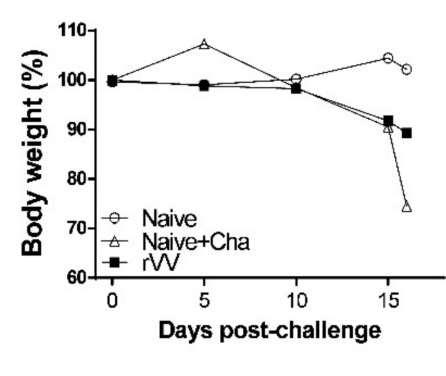

C

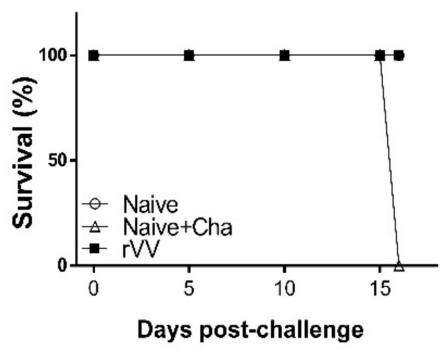

Figure 7. The ROP4-rVV vaccine protects mice against T. gondii ME49. The protective efficacy of the vaccine was evaluated by assessing cyst counts, bodyweight reduction, and survival. Cysts were counted from the right hemispheres of mice ( $n=6$ per group) under the microscope (A). Mice were monitored daily to check changes in bodyweight (B) and survival (C). Data are presented as mean \pm SD from 3 independent experiments and asterisks indicate statistical differences between groups $(* * *<0.001)$.

\section{Discussion}

Oral vaccines are highly desired for several reasons. They are easy to administer with excellent patient compliance since pain infliction is virtually non-existent. Since these vaccines do not require the presence of highly trained medical personnel for proper administration, it is an efficient mass vaccination strategy [19]. Nevertheless, the major obstacle with mucosal immunization via the oral route is the need to traverse through the highly acidic environment of the stomach. Consequently, antigenic epitopes are prone to degradation and this substantially lessens the efficacy of the vaccines [19]. While the ROP4-rVV vaccine used in the present study was subjected to such detrimental effects, they were immunogenic and conferred protection against a lethal dose of T. gondii ME49 challenge infection in mice.

Most of the immunological findings reported here are similar to the protective efficacy induced by ROP4-rBV vaccines as described in our previous study, although several differences in immunological parameters were noted [10]. Consistent with our previous study, a remarkable increase in GC B cells was observed from both the spleens and MLNs of mice. However, discrepancies were observed in the T cell populations. In the oral ROP4rBV immunized mice, differences were not significant compared to the control groups, while such differences were observed in the ROP4-rVV immunized mice. Differences were also detected in terms of brain inflammatory cytokine production. While significant IL-6 reductions were not observed in our earlier works with the ROP4-rBVs, drastic IL-6 and IFN- $\gamma$ reductions were observed in the current study. Although cyst counts were comparable for the two vectored vaccines in our works, bodyweight reductions were less severe when mice were immunized with the ROP4-rVVs. Given these results, the protection induced by the ROP4-rVV vaccine appears to be superior to that of the rBV vaccine expressing an identical antigen, thereby suggesting that $\mathrm{rVV}$ vectors serve as potent inducers of mucosal immunity in mice. In support of this notion, one study comparing the efficacies of MVA-based vaccines against $\mathrm{rBV}$ vaccines has reported similar findings. MVA vaccines expressing the VP2 antigen of African horse sickness virus conferred better protection in mice than $\mathrm{rBV}$ vaccines expressing the same antigen at an identical dosage and number of immunizations [20].

Antibodies are functionally required for mounting optimal protection against pathogens. Our findings revealed that immunization with the ROP4-rVV elicited significantly enhanced antibody responses in sera and also in the mucosal tissues. Mucosal antibodies, particularly the IgA subclass, are of importance. These antibodies deter the attachment of 
microorganisms to the mucosal surface [21]. Since the intestinal epithelial cells are the site of infection for T. gondii, mounting a strong first line of defense in these regions is important. The presence of these vaccine-induced mucosal antibodies, especially IgA, is crucial as they will act to limit parasitic entry into the host cells. Evidently, T. gondii-specific secretory IgA antibodies collected from patients were reported to have successfully inhibited the T. gondii infection of enterocytes [22]. As exemplified using these clinical samples, the ROP4-specific mucosal IgA elicited by the ROP4-rVV vaccine may have protected mice against challenge infection by limiting the parasitic entry into intestinal epithelial cells. Similarly, pro-inflammatory cytokines such as IFN- $\gamma$ are essential for resistance against $T$. gondii, as their production by $\mathrm{T}$ cells is required to inhibit tachyzoite proliferation in the brain $[23,24]$. Nevertheless, this comes at the expense of neuronal damage. For instance, in pneumococcal meningitis mice models, reduced neuronal damages were observed from IFN- $\gamma$ ablated mice whereas brain pathologies were much more severe in the wild-type mice [25]. Therefore, a balance in inflammatory cytokines that enable parasitic inhibition while inflicting minimal neuronal damage is necessary.

There were several discrepancies between our findings and those of another study. Surprisingly, immunizing C57BL/6 mice with the recombinant vaccinia virus vaccine (LC16m0 strain) expressing the GRA4 antigen of T. gondii failed to elicit antigen-specific antibody responses even after 3 immunizations at 3-week intervals [4]. Contrary to these results, profound antibody responses were observed in the sera and mucosal tissues of the ROP4-rVV immunized mice. Similar to our results, mice immunized with the ROP2-expressing recombinant MVA vaccine also developed high titers of antigen-specific antibody responses [3]. Identifying the exact factor accountable for such differences is unknown and difficult to discern, but it is possible that antigen candidates and the strain of the vaccinia virus may be involved. LC16m8 strain, which is derived from the LC16m0 strain, is categorized under 3rd generation vaccinia virus vaccines along with the MVA strain. However, their method of production and the extent of their attenuation greatly differs. Consequently, different strains of these 3rd generation vaccines were reported to possess differing virus properties, which include replication competency in mammalian cells, pro-inflammatory gene induction, and others.

Vaccinia virus dissemination into the ovaries of infected mice following intravenous and intraperitoneal inoculation has been reported, but such a phenomenon was not observed when the viruses were injected via a subcutaneous route [26]. Intranasally inoculating vaccinia virus resulted in uncontrolled virus replication in the murine lungs, which ultimately led to their death [27]. However, such issues were not observed from our study or by others [28] when vaccinia viruses were inoculated via oral routes, thus implying that the oral route of administration could be an effective way for developing vaccinia virus-based vaccine candidates. As previously mentioned, antigen degradation and efficacy loss is one detriment associated with oral vaccines. To overcome this issue and improve the efficacy of orally administered mucosal vaccines, alternative strategies could be employed. One such method would be immunization through the sublingual/buccal tissues outlining the oral cavity. Evidently, sublingual/buccal immunization with the MVA-based human immunodeficiency virus 1 (HIV-1) elicited potent mucosal immune responses and protected rhesus macaques upon challenge infection [29]. Another strategy would be to employ heterologous immunization as described through earlier MVA-based T. gondii vaccine studies $[4,5]$.

One major limitation of this study is the lack of data confirming long-term protection. In our study, all mice were sacrificed at an identical time point to enable accurate comparison of immunological parameters between groups. Thus, long-term protection could not be evaluated. Therefore, interpreting the findings illustrated in Figure $7 \mathrm{~B}$ would be speculative. For example, based on the downward trend of bodyweight, mice could either recover and survive for months or succumb to death via delayed disease progression. As such, further studies assessing this must be conducted. 


\section{Conclusions}

In summary, we demonstrated that orally immunizing mice with the ROP4-rVV vaccine induces robust systemic and mucosal immunity that fully protects mice from lethal challenge infection with $T$. gondii ME49. The live attenuated vaccine design strategy presented in this study may be suitable for the development of a safe and efficacious $T$. gondii vaccine.

Author Contributions: Conceptualization, F.-S.Q.; methodology, K.-W.Y. and F.-S.Q.; validation, K.W.Y.; formal analysis, K.-W.Y.; investigation, K.-W.Y., H.-J.K., M.-J.K., and G.-D.E.; resources, F.-S.Q.; data curation, K.-W.Y.; writing-original draft preparation, K.-W.Y. and K.-B.C.; writing-review and editing, K.-B.C. and F.-S.Q.; visualization, K.-W.Y.; supervision, F.-S.Q.; project administration, F.-S.Q.; funding acquisition, F.-S.Q. All authors have read and agreed to the published version of the manuscript.

Funding: This research was funded by the National Research Foundation of Korea (NRF) (2018R1A6A1 A03025124), the Ministry of Health \& Welfare, Republic of Korea (HV20C0085, HV20C0142).

Institutional Review Board Statement: This study was conducted according to the guidelines set by Kyung Hee University Institutional Animal Care and Use Committee (IACUC) and all experimental protocols involving animals have been approved (Permit number: KHUASP (SE) 20-648).

Informed Consent Statement: Not applicable.

Data Availability Statement: Data supporting the findings of this study are contained within the article.

Conflicts of Interest: The funders had no role in the design of the study; in the collection, analyses, or interpretation of data; in the writing of the manuscript; or in the decision to publish the results.

\section{References}

1. Montazeri, M.; Mehrzadi, S.; Sharif, M.; Sarvi, S.; Tanzifi, A.; Aghayan, S.A.; Daryani, A. Drug Resistance in Toxoplasma gondii. Front. Microbiol. 2018, 9, 2587. [CrossRef] [PubMed]

2. Rémy, V.; Zöllner, Y.; Heckmann, U. Vaccination: The cornerstone of an efficient healthcare system. J. Mark. Access Health Policy 2015, 3, 27041. [CrossRef]

3. Roque-Reséndiz, J.L.; Rosales, R.; Herion, P. MVA ROP2 vaccinia virus recombinant as a vaccine candidate for toxoplasmosis. Parasitology 2004, 128, 397-405. [CrossRef]

4. Zhang, G.; Huong, V.T.; Battur, B.; Zhou, J.; Zhang, H.; Liao, M.; Kawase, O.; Lee, E.G.; Dautu, G.; Igarashi, M.; et al. A heterologous prime-boost vaccination regime using DNA and a vaccinia virus, both expressing GRA4, induced protective immunity against Toxoplasma gondii infection in mice. Parasitology 2007, 134, 1339-1346. [CrossRef] [PubMed]

5. Mendes, É.A.; Fonseca, F.G.; Casério, B.M.; Colina, J.P.; Gazzinelli, R.T.; Caetano, B.C. Recombinant vaccines against T. gondii: Comparison between homologous and heterologous vaccination protocols using two viral vectors expressing SAG1. PLoS ONE 2013, 8, e63201. [CrossRef]

6. $\quad$ El Hajj, H.; Lebrun, M.; Arold, S.T.; Vial, H.; Labesse, G.; Dubremetz, J.F. ROP18 is a rhoptry kinase controlling the intracellular proliferation of Toxoplasma gondii. PLoS Pathog. 2007, 3, e14. [CrossRef] [PubMed]

7. Dubremetz, J.F. Rhoptries are major players in Toxoplasma gondii invasion and host cell interaction. Cell. Microbiol. 2007, 9 , 841-848. [CrossRef]

8. Kang, H.J.; Lee, S.H.; Kim, M.J.; Chu, K.B.; Lee, D.H.; Chopra, M.; Choi, H.J.; Park, H.; Jin, H.; Quan, F.S. Influenza Virus-Like Particles Presenting both Toxoplasma gondii ROP4 and ROP13 Enhance Protection against T. gondii Infection. Pharmaceutics 2019, 11, 342. [CrossRef]

9. Gatkowska, J.; Wieczorek, M.; Dziadek, B.; Dzitko, K.; Dziadek, J.; Długońska, H. Assessment of the antigenic and neuroprotective activity of the subunit anti-Toxoplasma vaccine in T. gondii experimentally infected mice. Vet. Parasitol. 2018, 254, 82-94. [CrossRef]

10. Yoon, K.W.; Chu, K.B.; Kang, H.J.; Kim, M.J.; Eom, G.D.; Lee, S.H.; Moon, E.K.; Quan, F.S. Mucosal Administration of Recombinant Baculovirus Displaying Toxoplasma gondii ROP4 Confers Protection Against T. gondii Challenge Infection in Mice. Front. Cell. Infect. Microbiol. 2021, 11, 735191. [CrossRef]

11. Tuboly, T.; Nagy, E.; Derbyshire, J.B. Potential viral vectors for the stimulation of mucosal antibody responses against enteric viral antigens in pigs. Res. Vet. Sci. 1993, 54, 345-350. [CrossRef]

12. van Oers, M.M. Vaccines for viral and parasitic diseases produced with baculovirus vectors. Adv. Virus Res. 2006, 68, 193-253 [CrossRef]

13. Tani, H.; Limn, C.K.; Yap, C.C.; Onishi, M.; Nozaki, M.; Nishimune, Y.; Okahashi, N.; Kitagawa, Y.; Watanabe, R.; Mochizuki, R.; et al. In vitro and in vivo gene delivery by recombinant baculoviruses. J. Virol. 2003, 77, 9799-9808. [CrossRef] 
14. Debnath, N.; Thakur, M.; Khushboo; Negi, N.P.; Gautam, V.; Kumar Yadav, A.; Kumar, D. Insight of oral vaccines as an alternative approach to health and disease management: An innovative intuition and challenges. Biotechnol. Bioeng. 2022, 119, 327-346. [CrossRef]

15. Blasco, R.; Moss, B. Selection of recombinant vaccinia viruses on the basis of plaque formation. Gene 1995, 158, 157-162. [CrossRef]

16. Kang, S.M.; Quan, F.S.; Huang, C.; Guo, L.; Ye, L.; Yang, C.; Compans, R.W. Modified HIV envelope proteins with enhanced binding to neutralizing monoclonal antibodies. Virology 2005, 331, 20-32. [CrossRef] [PubMed]

17. Kang, H.J.; Chu, K.B.; Kim, M.J.; Park, H.; Jin, H.; Lee, S.H.; Moon, E.K.; Quan, F.S. Evaluation of CpG-ODN-Adjuvanted Toxoplasma Gondii Virus-Like Particle Vaccine upon One, Two, and Three Immunizations. Pharmaceutics 2020, 12, 989. [CrossRef] [PubMed]

18. Kang, H.J.; Lee, S.H.; Chu, K.B.; Lee, D.H.; Quan, F.S. Virus-Like Particles Expressing Toxoplasma gondii Rhoptry Protein 18 Induces Better Protection Than Rhoptry Protein 4 against T. gondii Infection. Korean J. Parasitol. 2018, 56, 429-435. [CrossRef] [PubMed]

19. Lycke, N. Recent progress in mucosal vaccine development: Potential and limitations. Nat. Rev. Immunol. 2012, 12, 592-605. [CrossRef] [PubMed]

20. Aksular, M.; Calvo-Pinilla, E.; Marín-López, A.; Ortego, J.; Chambers, A.C.; King, L.A.; Castillo-Olivares, J. A single dose of African horse sickness virus (AHSV) VP2 based vaccines provides complete clinical protection in a mouse model. Vaccine 2018, 36, 7003-7010. [CrossRef]

21. Woof, J.M.; Mestecky, J. Mucosal immunoglobulins. Immunol. Rev. 2005, 206, 64-82. [CrossRef]

22. Mack, D.G.; McLeod, R. Human Toxoplasma gondii-specific secretory immunoglobulin A reduces T. gondii infection of enterocytes in vitro. J. Clin. Investig. 1992, 90, 2585-2592. [CrossRef] [PubMed]

23. Brown, C.R.; Hunter, C.A.; Estes, R.G.; Beckmann, E.; Forman, J.; David, C.; Remington, J.S.; McLeod, R. Definitive identification of a gene that confers resistance against Toxoplasma cyst burden and encephalitis. Immunology 1995, 85, 419-428. [PubMed]

24. Suzuki, Y.; Sa, Q.; Gehman, M.; Ochiai, E. Interferon-gamma- and perforin-mediated immune responses for resistance against Toxoplasma gondii in the brain. Expert Rev. Mol. Med. 2011, 13, e31. [CrossRef] [PubMed]

25. Too, L.K.; Ball, H.J.; McGregor, I.S.; Hunt, N.H. The pro-inflammatory cytokine interferon-gamma is an important driver of neuropathology and behavioural sequelae in experimental pneumococcal meningitis. Brain Behav. Immun. 2014, 40, 252-268. [CrossRef] [PubMed]

26. Karupiah, G.; Coupar, B.; Ramshaw, I.; Boyle, D.; Blanden, R.; Andrew, M. Vaccinia virus-mediated damage of murine ovaries and protection by virus-expressed interleukin-2. Immunol. Cell Biol. 1990, 68 Pt 5, 325-333. [CrossRef]

27. Hayasaka, D.; Ennis, F.A.; Terajima, M. Pathogeneses of respiratory infections with virulent and attenuated vaccinia viruses. Virol. J. 2007, 4, 22. [CrossRef]

28. Scheckelhoff, M.R.; Telford, S.R.; Hu, L.T. Protective efficacy of an oral vaccine to reduce carriage of Borrelia burgdorferi (strain N40) in mouse and tick reservoirs. Vaccine 2006, 24, 1949-1957. [CrossRef]

29. Jones, A.T.; Shen, X.; Walter, K.L.; LaBranche, C.C.; Wyatt, L.S.; Tomaras, G.D.; Montefiori, D.C.; Moss, B.; Barouch, D.H.; Clements, J.D.; et al. HIV-1 vaccination by needle-free oral injection induces strong mucosal immunity and protects against SHIV challenge. Nat. Commun. 2019, 10, 798. [CrossRef] 